\title{
Coherent spin ratchets: A spin-orbit based quantum ratchet mechanism for spin-polarized currents in ballistic conductors
}

\author{
Matthias Scheid \\ Institut für Theoretische Physik, Universität Regensburg, D-93040 Regensburg, Germany \\ Andreas Pfund \\ Institut für Theoretische Physik, Universität Regensburg, D-93040 Regensburg, Germany \\ and Solid State Physics Laboratory, ETH Hönggerberg, HPF E6, Zürich CH-8093, Switzerland \\ Dario Bercioux* \\ Institut für Theoretische Physik, Universität Regensburg, D-93040 Regensburg, Germany \\ and Physikalisches Institut, Albert-Ludwigs-Universität, D-79104 Freiburg, Germany \\ Klaus Richter \\ Institut für Theoretische Physik, Universität Regensburg, D-93040 Regensburg, Germany \\ (Received 16 May 2007; revised manuscript received 17 July 2007; published 2 November 2007)
}

\begin{abstract}
We demonstrate that the combined effect of a spatially periodic potential, lateral confinement, and spin-orbit interaction gives rise to a quantum ratchet mechanism for spin-polarized currents in two-dimensional coherent conductors. Upon adiabatic ac driving, in the absence of a net static bias, the system generates a directed spin current while the total charge current is zero. We analyze the underlying mechanism by employing symmetry properties of the scattering matrix and numerically verify the effect for different setups of ballistic conductors. The spin current direction can be changed upon tuning the Fermi energy or the strength of the Rashba spin-orbit coupling.
\end{abstract}

DOI: 10.1103/PhysRevB.76.195303

PACS number(s): 73.23.-b, 05.60.Gg, 72.25.-b

\section{INTRODUCTION}

Charge transport is usually studied by considering current in response to an externally applied bias. However, there has been growing interest throughout the last decade in mechanisms enabling directed particle motion in nanosystems without applying a net dc bias. In this respect, ratchets, periodic structures with broken spatial symmetry, e.g., saw tooth type potentials, represent a prominent class. Ratchets in the original sense are devices operating far from equilibrium by converting thermal fluctuations into directed particle transport in the presence of unbiased time-periodic driving. ${ }^{1}$ First discovered in the context of (overdamped) classical Brownian motion, ${ }^{2,3}$ the concept of dissipative ratchets was later generalized to the quantum realm. ${ }^{4}$ More recently, coherent ratchets and rectifiers have gained increasing attention. They are characterized by coherent quantum dynamics in the central periodic system in between leads where dissipation takes place. Proposals comprise molecular wires ${ }^{5}$ and cold atoms in optical lattices, ${ }^{6}$ besides Hamiltonian ratchets. ${ }^{7}$ Experimentally, ratchet-induced charge flow in the coherent regime was first observed in a chain of triangular-shaped lateral quantum $\operatorname{dots}^{8}$ and later in lateral superlattices. ${ }^{9}$

Here, we propose a different class of ratchet devices, namely, spin ratchets which act as sources for spin currents with simultaneously vanishing charge, respectively, particle currents. To be definite, we consider coherent transport through ballistic mesoscopic conductors in the presence of spin-orbit (SO) interaction. ${ }^{10}$ Contrary to particle ratchets, which rely on asymmetries in either the spatially periodic modulation or the time-periodic driving, a SO-based ratchet works even for symmetric periodic potentials. As possible realizations, we have in mind semiconductor heterostructures with Rashba SO interaction ${ }^{11}$ that can be tuned in strength by an external gate voltage allowing us to control the spin evolution.

Among other features, it is this property which is triggering recent broad interest in semiconductor-based spin electronics. ${ }^{12}$ Also, since direct spin injection from a ferromagnet into a semiconductor remains problematic, ${ }^{13}$ alternatively, several suggestions have been made for generating spin-polarized charge carriers without using magnets. In this respect, spin pumping appears promising, i.e., the generation of spin-polarized currents at zero bias via cyclic variation of at least two parameters. Different theoretical proposals based on $\mathrm{SO}^{14}$ and Zeeman ${ }^{15}$ mediated spin pumping in nonmagnetic semiconductors have been put forward ${ }^{16}$ and, in the latter case, experimentally observed in mesoscopic cavities. ${ }^{17}$

While pumps and ratchets share the appealing property of generating directed flow without net bias, ratchet transport requires only a single driving parameter. The periodic ratchet potential has a strong collective effect on the spin current and gives rise to distinct features such as spin current reversals upon parameter changes.

\section{MODEL AND SYMMETRY CONSIDERATIONS}

We consider a two-dimensional coherent ballistic conductor in the plane $(x, z)$ connected to two nonmagnetic leads. The Hamiltonian of the central system in presence of Rashba SO interaction reads 


$$
\mathcal{H}_{c}=\frac{\hat{p}^{2}}{2 m^{*}}+\frac{\hbar k_{\mathrm{SO}}}{m^{*}}\left(\hat{\sigma}_{x} \hat{p}_{z}-\hat{\sigma}_{z} \hat{p}_{x}\right)+U(x, z) .
$$

Here, $m^{*}$ is the effective electron mass, $U(x, z)$ includes the ratchet potential in the $x$ and a lateral transverse confinement in the $z$ direction, and $\hat{\sigma}_{i}$ denote Pauli spin matrices. The effect of the SO coupling with strength $k_{\text {SO }}$ is twofold: it leads to spin precession and it couples transversal modes in the confining potential. ${ }^{18}$

In view of a ratchet setup, we consider an additional timeperiodic driving term $\mathcal{H}_{V}(t)$ due to an external bias potential $V(t)$ with zero net bias (rocking ratchet). We study adiabatic driving (such that the system can adjust to the instantaneous equilibrium state), assuming that the driving period $t_{0}$ is large compared to the relevant time scales for transmission. This is the case in related experiments. ${ }^{8}$ The entire Hamiltonian then reads

$$
\mathcal{H}=\mathcal{H}_{c}+\mathcal{H}_{V}(t), \quad \mathcal{H}_{V}(t)=V(t) g(x, z ; V),
$$

where $g(x, z ; V)$ describes the spatial distribution of the voltage drop and should, in principle, be obtained selfconsistently from the particle density.

We model spin-dependent transport within a scattering approach assuming that inelastic processes take place only in the reservoirs. Then, the probability amplitude for an electron to pass through the conductor is given by the scattering matrix $\mathcal{S}_{n \sigma ; n^{\prime} \sigma^{\prime}}(E, V)$, where $n^{\prime}, n$ denote transverse modes and $\sigma^{\prime}, \sigma= \pm 1$ the spin directions in the incoming and outgoing leads, respectively. Making use of the unitarity of the scattering matrix, $\mathcal{S S}^{\dagger}=\mathcal{S}^{\dagger} \mathcal{S}=1$, and summing over all open channels in the left $(\mathrm{L})$ and right $(\mathrm{R})$ leads, respectively, we find the relations

$$
\sum_{\substack{n, \sigma \in \mathrm{R} \\ n^{\prime}, \sigma^{\prime} \in \mathrm{R} \cup \mathrm{L}}}\left|\mathcal{S}_{n, \sigma ; n^{\prime}, \sigma^{\prime}}\right|^{2}=\sum_{n, \sigma \in \mathrm{R}} 1, \sum_{\substack{n, \sigma \in \mathrm{R} \\ n^{\prime}, \sigma^{\prime} \in \mathrm{R} \cup \mathrm{L}}} \sigma\left|\mathcal{S}_{n, \sigma ; n^{\prime}, \sigma^{\prime}}\right|^{2}=0 .
$$

For further analysis, we consider an unbiased square wave driving $V(t)=V_{0} \operatorname{sgn}\left[\sin \left(2 \pi t / t_{0}\right)\right]$, restricted to the values $\pm V_{0}\left(V_{0}>0\right)$; generalizations to, e.g., harmonic driving are straightforward. The ratchet current is then given by the average of the steady-state currents in the two opposite rocking situations, $\left\langle I\left(V_{0}\right)\right\rangle=\left[I\left(+V_{0}\right)+I\left(-V_{0}\right)\right] / 2$, which we compute within the Landauer formalism relating conductance to transmission.

Contrary to charge current, spin current is usually not conserved. Thus, it is crucial to fix the measuring point, which we choose to be inside the right lead. Then, in view of Eq. (3), the ratchet charge $\left\langle I_{C}\right\rangle$ and spin $\left\langle I_{S}\right\rangle$ currents can be expressed as

$$
\left\langle I_{C / S}\left(V_{0}\right)\right\rangle=G_{C / S} \int_{E_{C}}^{\infty} d E \Delta f\left(E, V_{0}\right) \Delta T_{C / S}\left(E, V_{0}\right) .
$$

Here, the prefactor $G_{C / S}$ is equal to $e / 2 h$ for the charge current and $1 / 8 \pi$ for the spin current. $E_{C}$ denotes the energy of the conduction band edge, $\Delta f\left(E, V_{0}\right)=\left[f\left(E, E_{F}+V_{0} / 2\right)\right.$
$\left.-f\left(E, E_{F}-V_{0} / 2\right)\right]$ is the difference between the Fermi functions in the leads, and

$$
\Delta T_{C / S}\left(E, V_{0}\right)=T_{C / S}\left(E,+V_{0}\right)-T_{C / S}\left(E,-V_{0}\right) .
$$

With $T_{\sigma, \sigma^{\prime}}=\sum_{n \in \mathrm{R}, n^{\prime} \in \mathrm{L}}\left|\mathcal{S}_{n, \sigma ; n^{\prime}, \sigma^{\prime}}\right|^{2}$, the transmission probabilities for charge and spin in Eq. (5) are defined as

$$
\begin{gathered}
T_{C}(E, V)=\sum_{\substack{\sigma^{\prime}= \pm 1 \in \mathrm{L} \\
\sigma= \pm 1 \in \mathrm{R}}} T_{\sigma, \sigma^{\prime}}(E, V), \\
T_{S}(E, V)=\sum_{\sigma^{\prime}= \pm 1 \in \mathrm{L}}\left[T_{+, \sigma^{\prime}}(E, V)-T_{-, \sigma^{\prime}}(E, V)\right] .
\end{gathered}
$$

The latter is given by the difference between the transmission of spin-up and spin-down electrons upon exit, with the spin measured with respect to the $z$ axis.

Equation (5) indicates that $\Delta T_{C / S}\left(E, V_{0}\right)$, and thereby the average conductance vanishes in the linear response limit $V_{0} \rightarrow 0$. In the following, we consider the nonlinear regime and devise a minimum model for a spin ratchet mechanism by assuming identical leads and a spatially symmetric potential $U(x, z)$ in Eq. (1). The total Hamiltonian (2) is then invariant under the symmetry operation $\hat{\mathcal{P}}=\hat{\mathcal{C}} \hat{R}_{x} \hat{R}_{V} \hat{\sigma}_{z}$, where $\hat{\mathcal{C}}$ is the operator of complex conjugation, $\hat{R}_{x}$ inverses the $x$ coordinate, and $\hat{R}_{V}$ changes the sign of the applied voltage $( \pm V \leftrightarrow \mp V)$. The action of $\hat{\mathcal{P}}$ on the scattering states is to switch between the two rocking situations and to exchange the leads, i.e., a mode index $n$ is replaced by its corresponding mode $\tilde{n}$. Moreover, incoming (outgoing) states are transformed into outgoing (incoming) states with complex conjugated amplitude. It is then straightforward to show that

$$
S_{n, \sigma ; n^{\prime}, \sigma^{\prime}}\left(E, \mp V_{0}\right)=\sigma \sigma^{\prime} S_{\widetilde{n}^{\prime}, \sigma^{\prime} ; \tilde{n}, \sigma}\left(E, \pm V_{0}\right),
$$

leading to a vanishing charge current $\left\langle I_{C}\left(V_{0}\right)\right\rangle$ and a simplified expression for the ratchet spin transmission [Eq. (5)]:

$$
\Delta T_{S}\left(E, V_{0}\right)=2\left[T_{+,-}\left(E,+V_{0}\right)-T_{-,+}\left(E,+V_{0}\right)\right] .
$$

\section{RATCHET MECHANISM: NUMERICAL RESULTS}

We illustrate the prediction for a ratchet spin current [Eq. (4) with Eq. (9)] by performing numerical calculations for the Hamiltonian [Eqs. (1) and (2)]. The amplitudes $\mathcal{S}_{n \sigma^{\prime} ; m \sigma}(E, V)$ are obtained by projecting the Green's function of the open ratchet system onto an appropriate set of asymptotic spinors defining incoming and outgoing channels. For the efficient calculation of the $\mathcal{S}$-matrix elements, a realspace discretization of the Schrödinger equation combined with a recursive algorithm for the Green's functions was implemented for spin-dependent transport. ${ }^{21,22}$

As a model for a spin ratchet, we consider a ballistic two-dimensional quantum wire of width $W$ with Rashba SO strength $k_{\mathrm{SO}}$ and a one-dimensional periodic modulation (period $L$ ) composed of a set of $N$ symmetric potential barriers $U(x)=U_{0}[1-\cos (2 \pi x / L)]$. We assume a linear voltage drop $^{19}$ across the system, $g(x, z)=1 / 2-x /(N L)$ in Eq. (2). To 


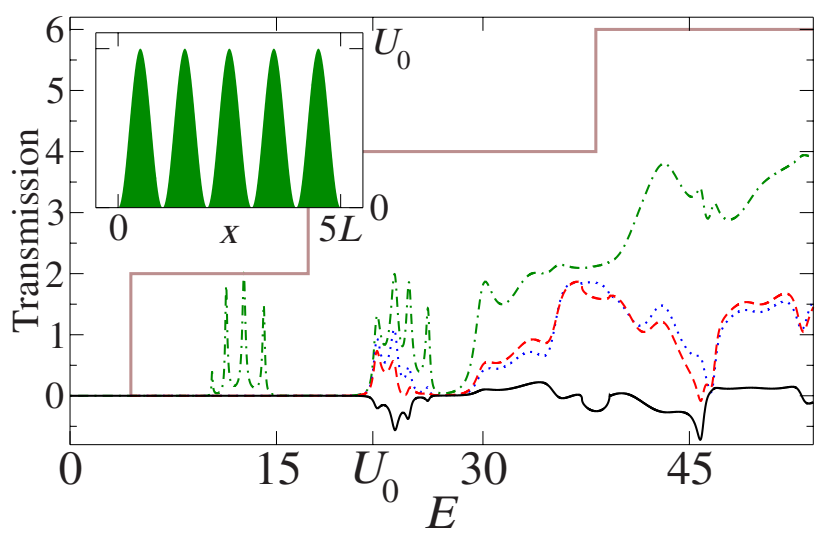

FIG. 1. (Color online) Spin-dependent transmissions as a function of the injection energy $E=(k L)^{2}$ in the presence of Rashba spin-orbit interaction $\left(k_{\mathrm{SO}} L=1.5\right)$ for a short periodic chain of five symmetrical potential barriers (see inset, barrier height $U_{0}=22$ ) and moderate rocking amplitude $V_{0}=2$. The dashed (red/gray) and dotted (blue/dark gray) lines indicate $T_{S}$, Eq. (7), in the two rocking situations. The solid (black) line depicts the ratchet spin transmission, Eq. (5), with the sign indicating the flow direction. For reference, the dashed-dotted (green/light gray) curve shows $T_{C}$, Eq. (6), and the staircase function $T_{C}$ for a wire without potential barriers and $\mathrm{SO}$ interaction.

simplify the assessment of the rich parameter space $\left(E_{F}, U(x), V, k_{\mathrm{SO}}, N\right)$ of the problem ( $L$ can be scaled out and $W$ is fixed to $1.5 L$ ) and to analyze the mechanisms for spin currents, we first consider a strip with $N=5$ potential barriers (see inset in Fig. 1) and few open transverse modes. Figure 1 shows the numerically obtained spin transmission probabilities $T_{S}(E, V)$, Eq. (7), for $k_{\mathrm{SO}} L=1.5$ in the two rocking situations $\pm V_{0}$ (dashed and dotted lines, respectively). The solid line represents the resulting ratchet spin transmission $\Delta T_{S}$, Eq. (5). For comparison, the dashed-dotted curve shows $T_{C}\left(+V_{0}\right)=T_{C}\left(-V_{0}\right)$, Eq. (6), and the staircase function the successive opening of transverse modes $n=1,2,3$ in the overall transmission of the conductor without potential barriers and $\mathrm{SO}$ interaction.
At energies below $U_{0}$ and within the first conducting transverse mode, the spin transmissions $T_{S}\left( \pm V_{0}\right)$ are zero, while the total transmission $T_{C}\left( \pm V_{0}\right)$ is suppressed up to a sequence of four peaks representing resonant tunneling through states which can be viewed as precursors of the lowest Bloch band in the limit of an infinite periodic potential. When the second mode is opened, spin polarization is possible (see model below) and takes different values in the two rocking situations leading to a finite ratchet spin transmission. Two transmission peak sequences, related to the lowest one, reappear at higher energies (around $E=24$ and 45), both for $T_{C}\left( \pm V_{0}\right)$ and for $T_{S}\left( \pm V_{0}\right)$, owing to corresponding resonant Bloch states involving the second and third transverse modes. The enhanced ratchet spin transmission at the opening of the third mode (at $E=38$ ) can be associated with a "classical" rectification effect resulting from a different number of open modes in one lead in the two rocking situations.

Figure 1 demonstrates moreover that the associated spin current changes sign several times upon variation of the energy, opening up the experimental possibility to control the spin current direction through the carrier density via an external gate. This energy dependence of the spin current also implies current inversion as a function of temperature. ${ }^{21}$ Such behavior is considered as typical for quantum (particle) ratchets. ${ }^{4,8}$

In Fig. 2(a), we present the ratchet spin transmission $\Delta T_{S}$ as a function of the barrier number $N$. Obviously, $\Delta T_{S}$ approaches different asymptotic values depending on the Fermi energy: For energies in resonance with the first Bloch band (lowest trace), $\Delta T_{S}$ exhibits a long- and a short-scale frequency oscillation owing to commensurability between the spin precession length $L_{\mathrm{SO}}=\pi / k_{\mathrm{SO}}$ and the geometry of the periodic system. For off-resonant injection energies, two characteristic, distinct behaviors are shown: a large-scale oscillation (upper curve) and a nearly constant behavior (middle trace), respectively. It is remarkable that in all cases, the periodic structure enhances considerably the absolute value of $\Delta T_{S}$.

In Fig. 2(b), we show the ratchet spin conductance, $\left\langle I_{S}\right\rangle$ $\times\left(e / V_{0}\right)$, as a function of the applied driving voltage for a
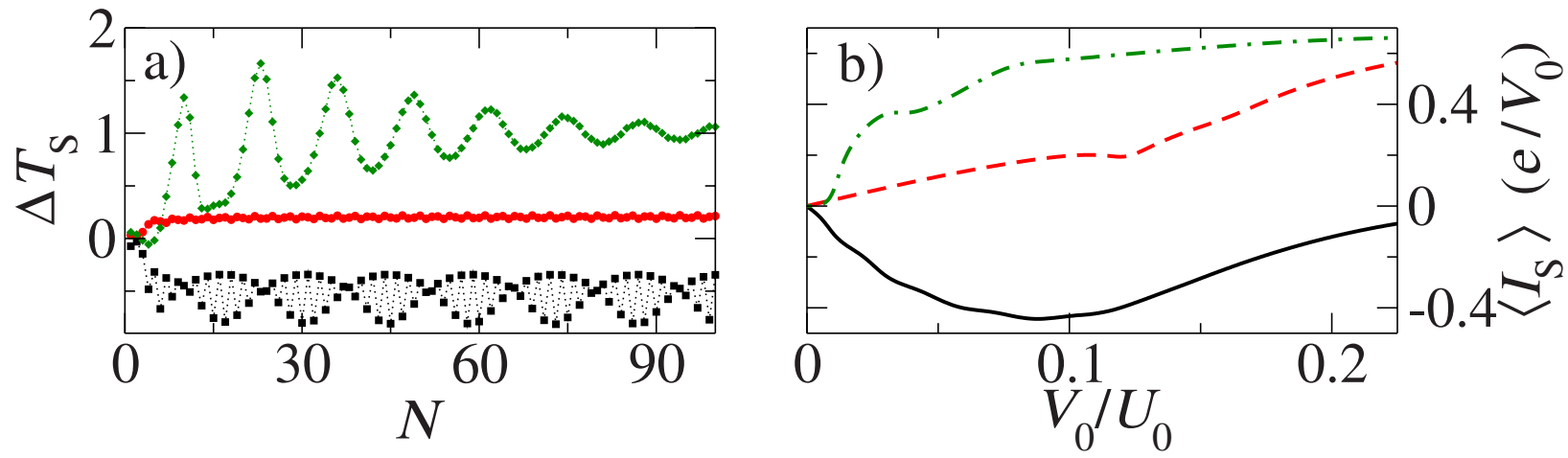

FIG. 2. (Color online) (a) Ratchet spin transmission as a function of the number of barriers $N$ for $k_{\mathrm{SO}} L=1.5, U_{0}=22, V_{0}=2$, and energies $E=(k L)^{2}=24$ (black symbols, lower line), 33 (red/gray, middle line), and 35.5 (green/light gray, upper line). (b) Ratchet spin conductance $\left\langle I_{S}\right\rangle\left(e / V_{0}\right)$ at zero temperature in units of $e G_{S}$ as a function of applied voltage $V_{0}$ for $N=20, k_{\mathrm{SO}} L=1.5, U_{0}=22$, and $E=24$ (black solid line), 33 (red/gray dashed line), and 35.5 (green/light gray dash-dotted line). 


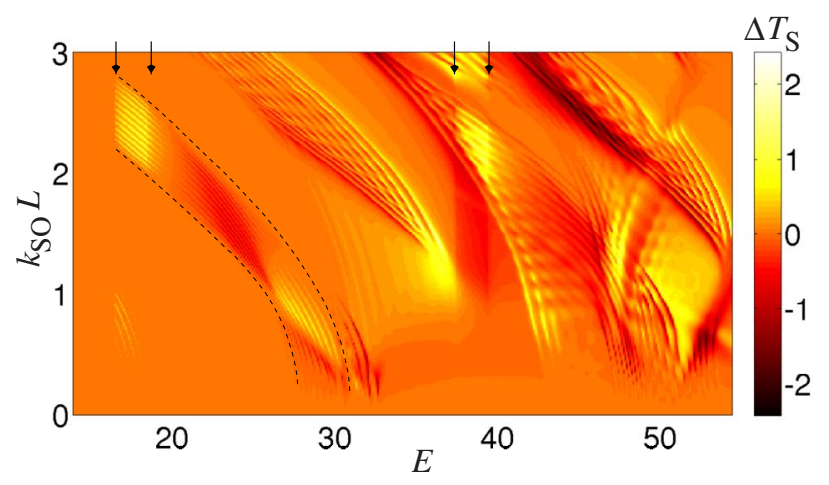

FIG. 3. (Color online) Ratchet spin transmission as a function of energy $E=(k L)^{2}$ and SO interaction $k_{\mathrm{SO}} L$ for $N=20, V_{0}=2$, and $U_{0}=22$. The dashed lines are guide to the eye for the shift of the first Bloch band.

system with 20 barriers. For energies within the first Bloch band (solid line), the ratchet spin conductance exhibits a nonmonotonic behavior. For the off-resonant cases (dashed and dashed-dotted line), it is monotonically increasing in the voltage window considered.

In Fig. 3, we present the ratchet spin transmission as a function of injection energy $E$ and Rashba SO interaction $k_{\text {SO. }}$. We find a rich structure in the explored parameter space, where both large positive and negative values of the ratchet spin transmission can be observed. In the whole energy range, peaks due to resonant tunneling are visible, which are shifted to lower energies for increasing SO coupling (e.g., region between dashed lines). Furthermore, we observe discontinuities in the spin transmission at energies where an additional transversal mode in one of the leads opens up (marked by arrows).

For InAs quantum wells, $L_{\mathrm{SO}}$ is of the order of $0.2 \mu \mathrm{m},{ }^{23}$ in InGaAs, it has been tuned from 0.7 to $1.6 \mu \mathrm{m},{ }^{24}$ and in GaAs from 2.3 to $5.6 \mu \mathrm{m} ;{ }^{25}$ the range of SO coupling $k_{\mathrm{SO}} L=\pi L / L_{\mathrm{SO}}$ given in Fig. 3 can be achieved in experiments for period $L$ on scales of micrometers. Spin-polarized currents as predicted here exceed those observed with experimental detection schemes, reported, e.g., in Ref. 17.

\section{RATCHET MECHANISM: SIMPLIFIED MODEL}

Finally, we present a simplified model providing additional insight into the underlying mechanism for the occurrence of a finite ratchet spin current. We consider a wire with two open transverse modes $(n=1,2)$ and a smooth symmetric potential barrier $U(x)$ in the two rocking situations, see Fig. 4. Upon adiabatically traversing the barrier from A via B to $\mathrm{C}$, the spin-orbit split energy spectrum $E_{n}\left(k_{x}\right)$ for electrons is shifted up and down. For fixed Fermi energy $E_{F}$, the initial shift causes a depopulation of the upper levels $(n=2)$ and a spin-dependent repopulation while moving from B to C. When $E_{F}$ is traversing an anticrossing between successive modes (see the region indicated by the dashed window in

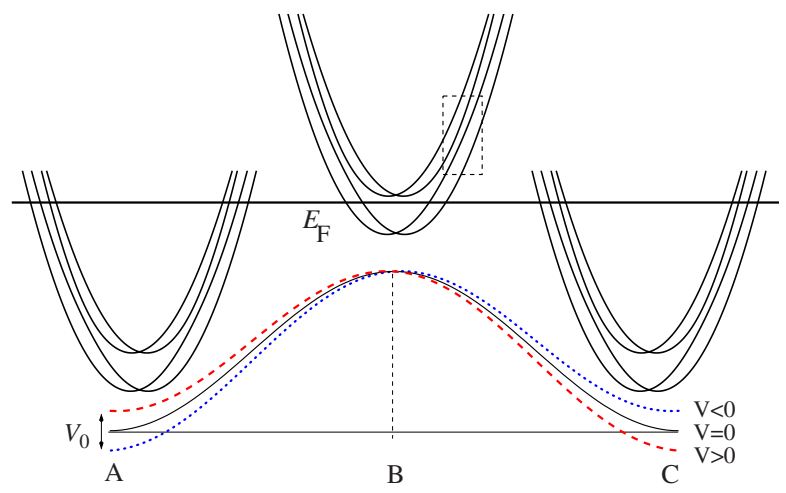

FIG. 4. (Color online) Illustration of the spin polarization mechanism for transmission through a strip with a single adiabatic symmetric potential barrier $U(x)$ (solid line) in the two rocking situations (dashed and dotted line). At points $\mathrm{A}, \mathrm{B}$, and $\mathrm{C}$, the position-dependent energy dispersion relation $E_{n}\left(k_{x}\right)$ is sketched with respect to the Fermi energy $E_{F}$ (horizontal line) for two transverse modes and SO-induced spin splitting of each mode.

Fig. 4), there is a certain probability $P$ for the electrons to change their spin state. This causes an asymmetry between spin-up and -down states for the repopulated levels. ${ }^{20}$ The related transition probability can be computed in a LandauZener picture and reads, for a transverse parabolic confinement of frequency $\omega_{0}$,

$$
P\left( \pm V_{0}\right)=1-\exp \left\{\frac{-\pi k_{\mathrm{SO}} \omega_{0} / \Sigma_{z}}{(\partial / \partial x)\left[U(x, z) \pm V_{0} g(x, z)\right]}\right\} .
$$

Here, $\Sigma_{z}$ denotes the difference in the polarizations of the two modes involved. The spin transmission is proportional to $P(V)$ and is thus different in the two rocking situations. Hence, the ratchet spin current $\left\langle I_{S}\left(V_{0}\right)\right\rangle$ is nonzero, even in the case of a symmetric barrier. Expanding Eq. (10) for small $V_{0}$ allows us to qualitatively understand the linear dependence of the ratchet spin conductance for small $V_{0}$ in Fig. 2. However, a quantitative explanation of the spin ratchet effect for a periodic, non-necessarily adiabatic potential is beyond this model.

\section{CONCLUSIONS}

The overall analysis indicates that the ratchet setup, carrying features of a spin rectifier, differs from the proposals $^{14,15,17}$ for spin pumps, since it operates with a single driving parameter and invokes quantum tunneling effects, and the spin transmission is governed by the spatial periodicity of the underlying potential. Further calculations ${ }^{21}$ for combined Rashba and Dresselhaus ${ }^{26} \mathrm{SO}$ coupling do not alter the overall picture but show that the spin current direction can be changed upon tuning the relative strength of the two coupling mechanisms.

To summarize, we showed that ratchets built from mesoscopic conductors with SO interaction generate spin currents in an experimentally accessible parameter regime. Many 
further interesting questions open up within this concept, including the exploration of spin ratchet effects for nonadiabatic driving and for dissipative and nonequilibrium particle and spin dynamics.

\section{ACKNOWLEDGMENTS}

We thank P. Hänggi, M. Grifoni, and M. Strehl for useful discussions and acknowledge support from the German Science Foundation (DFG) within SFB 689. *dario.bercioux@physik.uni-freiburg.de

${ }^{1}$ For recent overviews, see the special issue in 75 (2), 167 (2002), edited by H. Linke P. Reimann, Phys. Rep. 361, 57 (2002).

${ }^{2}$ P. Hänggi and R. Bartussek, in Nonlinear Physics of Complex Systems, edited by J. Parisi, S. C. Müller, and W. W. Zimmermann, Lecture Notes in Physics Vol. 476 (Springer, Berlin, 1996), p. 294.

${ }^{3}$ F. Jülicher, A. Adjari, and J. Prost, Rev. Mod. Phys. 69, 1269 (1997); R. D. Astumian, Science 276, 917 (1997).

${ }^{4}$ P. Reimann, M. Grifoni, and P. Hänggi, Phys. Rev. Lett. 79, 10 (1997).

${ }^{5}$ J. Lehmann, S. Kohler, P. Hänggi, and A. Nitzan, Phys. Rev. Lett. 88, 228305 (2002).

${ }^{6}$ E. Lundh and M. Wallin, Phys. Rev. Lett. 94, 110603 (2005).

${ }^{7}$ S. Flach, O. Yevtushenko, and Y. Zolotaryuk, Phys. Rev. Lett. 84, 2358 (2000); H. Schanz, M.-F. Otto, R. Ketzmerick, and T. Dittrich, ibid. 87, 070601 (2001).

${ }^{8}$ H. Linke, T. E. Humphrey, A. Löfgren, A. O. Sushkov, R. Newbury, R. P. Taylor, and P. Omling, Science 286, 2314 (1999).

${ }^{9}$ E. M. Höhberger, A. Lorke, W. Wegscheider, and M. Bichler, Appl. Phys. Lett. 78, 2905 (2001).

${ }^{10}$ For recent proposals of spin ratchets based on the Zeeman effect, see M. Scheid, M. Wimmer, D. Bercioux, and K. Richter, Phys. Status Solidi C 3, 4235 (2006); M. Scheid, D. Bercioux, and K. Richter, arXiv:0707.2478v1, New. J. Phys. (to be published).

${ }^{11}$ E. Rashba, Fiz. Tverd. Tela (Leningrad) 2, 1224 (1960) [Sov. Phys. Solid State 2, 1109 (1960)].

${ }^{12}$ I. Žutíc, J. Fabian, and S. Das Sarma, Rev. Mod. Phys. 76, 323 (2004).

${ }^{13}$ G. Schmidt, D. Ferrand, L. W. Molenkamp, A. T. Filip, and B. J. van Wees, Phys. Rev. B 62, R4790 (2000).
${ }^{14}$ P. Sharma and P. W. Brouwer, Phys. Rev. Lett. 91, 166801 (2003); M. Governale, F. Taddei, and R. Fazio, Phys. Rev. B 68, 155324 (2003).

${ }^{15}$ E. R. Mucciolo, C. Chamon, and C. M. Marcus, Phys. Rev. Lett. 89, 146802 (2002); M. Blaauboer and C. M. L. Fricot, Phys. Rev. B 71, 041303(R) (2005).

${ }^{16}$ Corresponding settings, where a precessing magnetization in a ferromagnet emits spin currents, were reviewed by Y. Tserkovnyak, A. Brataas, G. E. W. Bauer, and B. I. Halperin, Rev. Mod. Phys. 77, 1375 (2005).

${ }^{17}$ S. K. Watson, R. M. Potok, C. M. Marcus, and V. Umansky, Phys. Rev. Lett. 91, 258301 (2003).

${ }^{18}$ F. Mireles and G. Kirczenow, Phys. Rev. B 64, 024426 (2001); M. Governale and U. Zülicke, ibid. 66, 073311 (2002); C. A. Perroni, D. Bercioux, V. M. Ramaglia, and V. Cataudella, J. Phys.: Condens. Matter 19, 186227 (2007).

${ }^{19}$ An analysis (Ref. 21) of different models for the voltage drop shows that the results for the spin current, up to slight quantitative changes, are not altered qualitatively.

${ }^{20}$ M. Eto, T. Hayashi, and Y. Kurotani, J. Phys. Soc. Jpn. 74, 1934 (2005).

${ }^{21}$ D. Bercioux, M. Scheid, A. Pfund, and K. Richter (unpublished).

${ }^{22}$ D. Frustaglia, M. Hentschel, and K. Richter, Phys. Rev. Lett. 87, 256602 (2001); Phys. Rev. B 69, 155327 (2004).

${ }^{23}$ D. Grundler, Phys. Rev. Lett. 84, 6074 (2000).

${ }^{24}$ T. Bergsten, T. Kobayashi, Y. Sekine, and J. Nitta, Phys. Rev. Lett. 97, 196803 (2006).

${ }^{25}$ D. M. Zumbühl, J. B. Miller, C. M. Marcus, K. Campman, and A. C. Gossard, Phys. Rev. Lett. 89, 276803 (2002).

${ }^{26}$ G. Dresselhaus, Phys. Rev. 100, 580 (1955). 